\title{
Refresher Course in Applications of Quantum Mechanics to 'Atoms, Molecules and Radiation'
}

\author{
8- 20 December 2014 \\ at Indian Academy of Sciences, Bangalore \\ Sponsored by Indian Academy of Sciences, Bangalore, \\ Indian National Science Academy, New Delhi \\ The National Academy of Sciences, India, Allahabad
}

\begin{abstract}
A Refresher Course in Applications of Quantum Mechanics to 'Atoms, Molecules and Radiation' will be held at the Indian Academy of Sciences, Bangalore from December 8 to 20. 2014. The Course is primarily aimed at teachers teaching quantum mechanics and/ or atomic and molecular physics at the UG/PG level. The course will cover basics of quantum mechanics and its applications to atoms, molecules and their interaction with radiation, through lectures and tutorials. College/ university teachers having at least a Master's degree in Physics are particularly encouraged to apply.
\end{abstract}

Topics: Most of the topics in standard UG/ PG level courses in these areas will be covered. There will be four modules:

Module 1: Basics of quantum mechanics (review): Interpretative postulates, Schrodinger equation, abstract vector space formulation, Dirac notation, representations and pictures, linear oscillator, perturbation theory

Module 2: Orbital and spin angular momentum, addition of angular momenta, hydrogen atom, fine structure, helium atom, multi-electron atoms (brief), L-S and $\mathrm{j}$-j coupling.

Module 3: Quantum theory of radiation (non-covariant), spontaneous, stimulated emission and absorption probabilities, electric dipole selection rules, Zeeman effect

Module 4: Hydrogen molecule ion, molecular orbitals, diatomic molecules, nature of bonds, rotational and vibrational spectra, polyatomic molecules (brief)

Resource Persons: A. Rangwala (Univ. of Mumbai), Swapan K. Ghosh (BARC and Centre for Basic Sciences, Mumbai), Ushadevi (Bangalore Univ.), Arvind Kumar ( Centre for Basic Sciences: formerly at Homi Bhabha Centre for Science Education (TIFR), Mumbai).

Course Director: Arvind Kumar

Teachers who wish to participate in the course should send their applications online using the following link.http://web-japps.ias.ac.in:8080/Refreshcourse/RCQM.jsp

A print copy of the same application through the Head of the institution should be sent by speed post to: Sri G. Madhavan, Coordinator, Science Education Programmes, Indian Academy of Sciences C.V. Raman Avenue, Sadashivanagar, Bangalore 560080

For any further query, please e-mail to sep@ias.ernet.in

Please note that participants have to attend the full duration of the Course.

Last date for receipt of online applications: 20 September 2014. 